\title{
Whole-Exome Sequencing Reveals a Recurrent D401N Mutation in the COMP gene that Causes Multiple Epiphyseal Dysplasia
}

\author{
Jing Wang ${ }^{1}$, Yuxian Wang ${ }^{2}$, Meiling Chong ${ }^{3}$, Yaohong Hao ${ }^{4}$, Zhuoyu Li ${ }^{1}$, Changxin Wu ${ }^{1}$, and Han Xiao ${ }^{1 *}$ \\ ${ }^{1}$ Institutes of Biomedical Sciences, Key Laboratory of Chemical Biology and Molecular Engineering of National Ministry of Education, \\ Shanxi University, China
}

${ }^{2}$ Department of Obstetrics and Gynecology, The First Hospital Affiliated To Shanxi Medical University, China

${ }^{3}$ Veritas Genetics Asia Inc, China

${ }^{4}$ Caregeno Medical Laboratories, China

Received: 眥 April 25, 2018; Published: 眥 May 07, 2018

*Corresponding author: Han Xiao, Institutes of Biomedical Sciences, Key Laboratory of Chemical Biology and Molecular Engineering of National Ministry of Education, Shanxi University, China

\begin{abstract}
Multiple epiphyseal dysplasia (MED) is a rare osteochondrodysplasia characterized by moderate short limb dwarfism and earlyonset osteoarthrosis. By whole-exome sequencing (WES), we identified a dominantly inherited mutation (c.1201G>A; p.D401N) in cartilage oligomeric matrix protein (COMP) in a large four-generation Chinese family. Immunofluorescence analysis revealed mutant COMP secretion was severely impaired. Our result expands the mutational spectrum of COMP and provides strong evidence for the genotype-phenotype correlation of COMP pathogenicity in MED.
\end{abstract}

Keywords: MED; COMP; WES

Abbreviations: MED: Multiple Epiphyseal Dysplasia; WES: Whole-Exome Sequencing; GSDs: Genetic Skeletal Diseases

\section{Introduction}

Human genetic skeletal diseases (GSDs) are an extremely diverse and COMPlex group of rare genetic condition that primarily affect the development and homeostasis of the osseous skeleton. GSDs not only cause patients in pain and disability, but also bring poor quality of life and high healthcare costs. According to the 2015 Nosology and Classification of the GSDs, there are 436 wellcharacterized skeletal diseases that are classified primarily on the basis of clinical, radiographic, and molecular criteria [1,2].

Multiple epiphyseal dysplasia (MED/EDM1, MIM no.132400) is a rare GSDs disease affecting the development of epiphyses. The main clinical features include mild short stature, lower limb deformities and early onset osteoarthritis [3,4]. MED is genetically heterogeneous and can result from mutations in COMP, DTDST (SLC26A2), MATN3, COL9A1, COL9A2 or COL9A3 or CANT1 [5-7], with the majority of cases results from mutations in COMP gene. These mutations cause disorganized endochondral ossification of the epiphysis, ultimately leading to destruction of the articular cartilage.

COMP is a $524 \mathrm{kD}$ secreted pentameric extracellular glycoprotein found in cartilage, tendon, and synovium, and plays an essential role in maintaining the extracellular matrix structure in cartilage [8]. Multiple functions have been suggested for COMP, including regulation of collagen fibril assembly, chondrocytes proliferation and interactions with other matrix proteins such as collagens type II, IX and matrilin-3 (MATN3) [9,10]. COMP mutation can affect the secretion of COMP protein and cause its retention in the ER [11]. In the current study, we identify a recurrent pathogenic SNV in COMP gene that cause MED by WES in a four-generation Chinese family this mutation is further validated using Sanger Sequencing. We examined the effects of mutation (D401N) in Hela cells and found this mutation affects COMP protein secretion and causes its retention in the ER. 


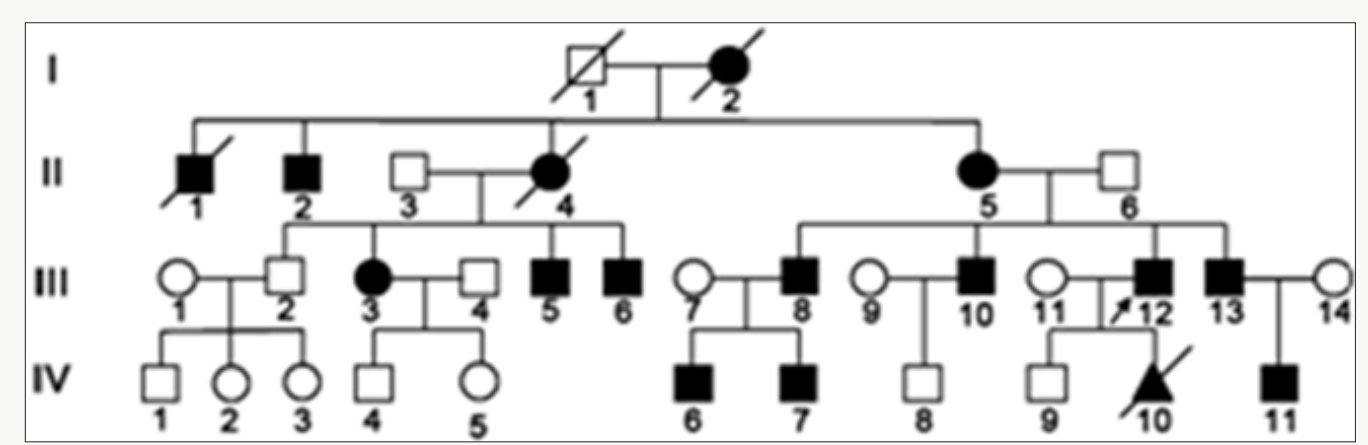

Figure 1A: Square indicates male, and circles indicate females. Blackened symbols denote affected individuals. The proband is indicated by arrows $(\nearrow)$.

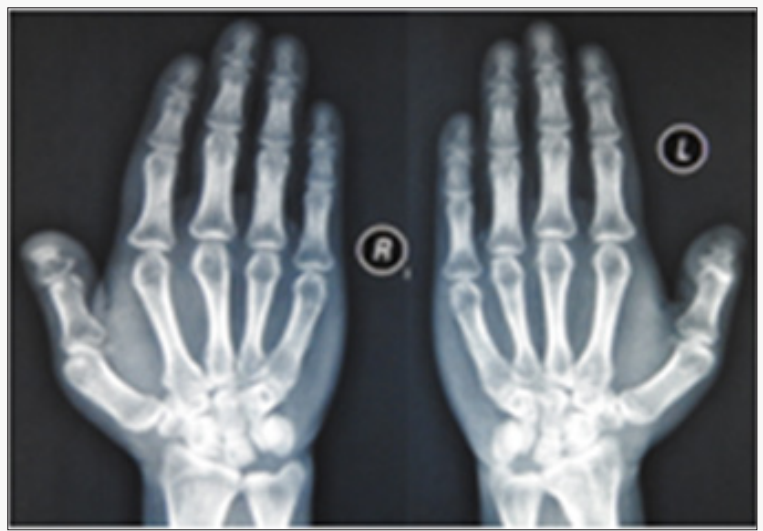

Figure 1B: Right hand and left hand X ray of the proband showed bilateral short and small phalanges, markedly irregularities of the distal radial and ulnar epiphyses.

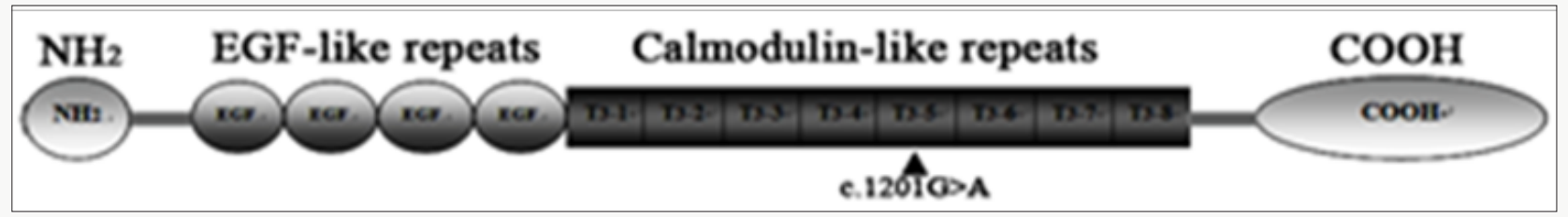

Figure 1C: The structure of a COMP monomer, showing the locations of the N-terminal domain (NH2), the EGF-like and calmodulin-like repeats, and the C-terminal domain $(\mathrm{COOH})$, is shown at the top.

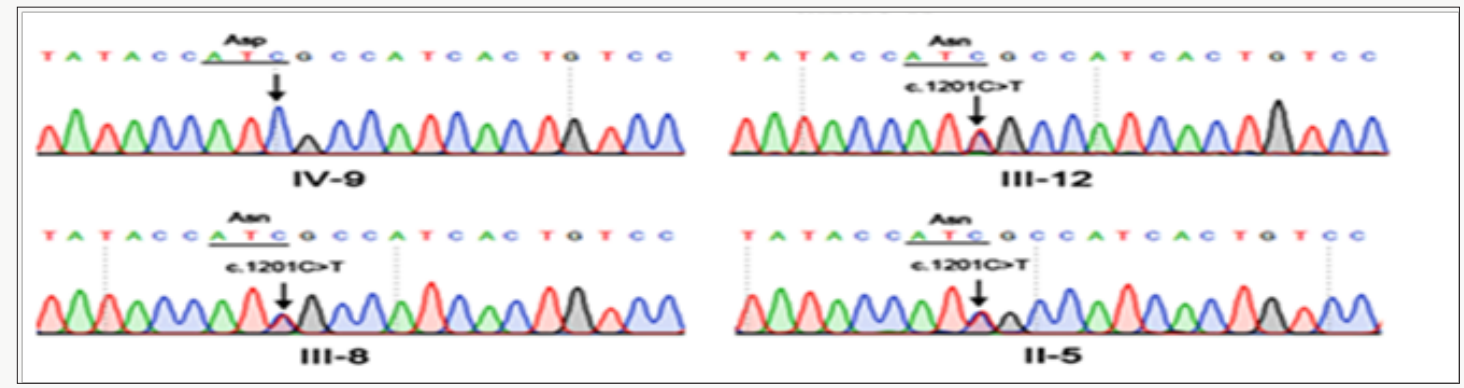

Figure 1D: Heterrogenous c.1201C > T (minus strand) was detected in patient III-12 111-8, 11-5, whilst this mutation is absent in healthy family members.

Figure 1: Family pedigree and sequencing results.

We investigated a four-generation Chinese family affected with MED (Figure 1A). Sixteen patients were included in the study; patients were identified and followed up for skeletal dysplasias at specialized clinics in participating institutions. Diagnosis of MED was made based on clinical and radiographic examinations, as described previously [12]. The proband had MED common signs: joint pain affecting the hip and knee joint, muscular hypotonic, joint laxity, mild genu vara. X-ray examination showed bilateral short and small phalanges, markedly irregularities of the distal radial and ulnar epiphyses (Figure 1B). In this family, all patients had the common features of MED. 
We obtained written informed consent to perform molecular studies, which were approved by Institutional Review Board of Shanxi Medical University. We performed WES on genomic DNA sample of the proband (III-12) of the Chinese Han family with MED in order to identify the causal genes. A total of 134,056 genetic variants, including 14990 non-synonymous changes, were occurred at the coding sequence or the canonical dinucleotide of the splice site junctions. Variants were functionally annotated and filtered using in-house cloud-based rare disease NGS analysis platform with builds in public databases (dbSNP, OMIM, ESP, Clinvar,
1,000 Genomes) as previously described [13]. Exonic sequence alterations and intronic variants at exon-intron boundaries, with unknown frequency or minor allele frequency (MAF) $<1 \%$ and not present in the homozygous state in those databases were retained. Subsequently, a missense heterozygous mutation in the COMP gene, with a $\mathrm{G}$ to A transition at position 1201, resulting in a substitution of aspartic acid for asparagines at amino acid position 401 (c.1201G>A; p.D401N), in the T3 motifs of COMP, was identified as the potential disease-causing gene of MED (Figure 1C).

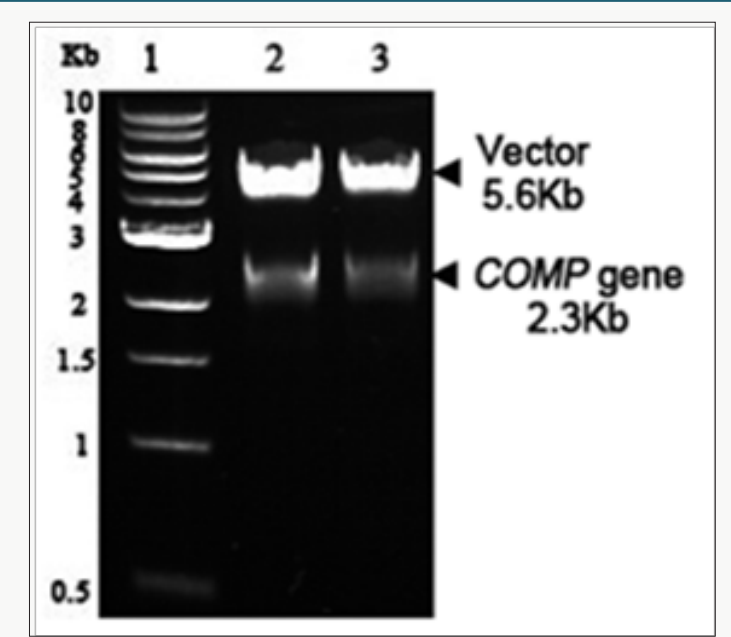

Figure 2A: COMP construct verification by double-digestion with AgeI/EcoRI. 1. Marker; 2. pcDNA-2Flag-WT-COMP; 3. pcDNA-2Flag-MT-COMP, harboring c.1201G>A mutation.

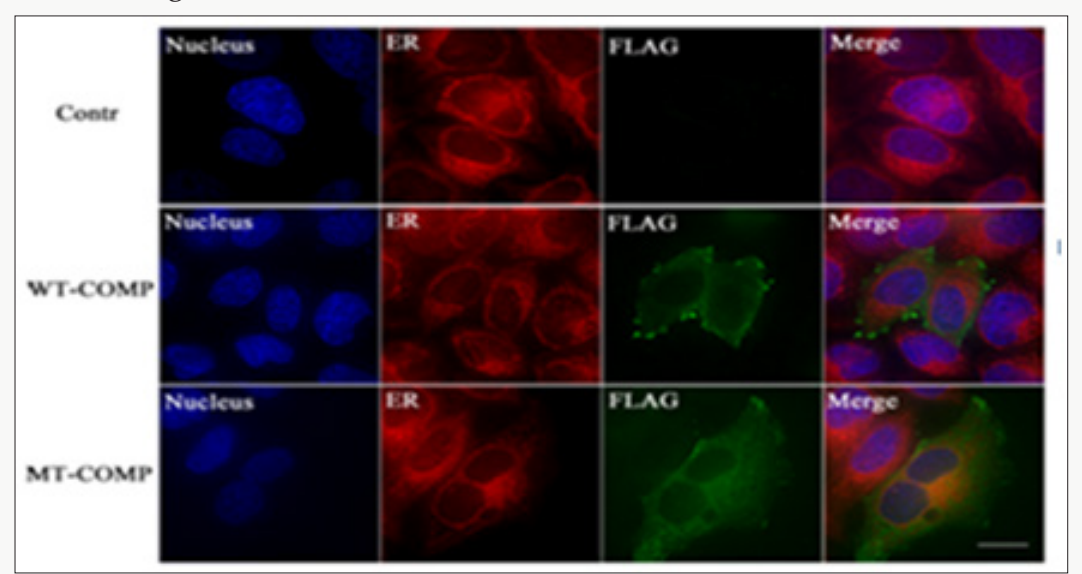

Figure 2B: Hela cells were transfect with WT-COMP and MT-COMP and cultured for 24 hours at $37^{\circ} \mathrm{C}$. The cells were fixed and incubated with the primary antibody (anti-FLAG) followed by FITC-labeled secondary antibodies. No COMP staining was detected in untransfected cells. Scale bar $=15 \mu \mathrm{m}$.

Figure 2: MT-COMP secretion was impaired.

To confirm the WES results, we performed Sanger sequencing for 11 family members to validate the potential disease causing variant. Sequencing results showed co-segregation of the pathogenic variant (c.1201G>A) with the disease phenotype (Figure 1D). Primers used for COMP causative variant validation were as follows: 11F: 5'-GAAGTCATTCTGGCCTGGTC-3' and 12R: $5^{\prime}$-GGTAGCCTTTGACAAACGCT-3'). To study the functional consequences of $\mathrm{D} 401 \mathrm{~N}$ mutation in COMP, we constructed the
FLAG-tagged full-length wild-type COMP (WT-COMP) and the mutant construct carrying D401N mutation, which was confirmed by Sanger sequencing that the correct mutation had been introduced and other PCR-generated errors were not incorporated (Figure2A). Immunofluorescence was performed using anti-FLAG antibody that specifically recognizes recombinant COMP (Figure 2B) and cytopainter ER staining kit to stain the ER. As shown in the merged images, at $24 \mathrm{~h}$ post-transfection, a significant portion of 
WT-COMP was localized to the cell surface; In contrast, MT-COMP protein was retained in the cytoplasm and co-localized with ER marker (red), suggesting that MT-COMP was retained in the ER and recapitulates the trafficking pathology in the mouse model of pseudoachondroplasia [14].

MED is a genetically heterogeneous disorder with marked clinical variability. Mutations in COMP gene is the most common form, accounting for at least half of the cases, with the majority of cases results from mutations in COMP gene and $85 \%$ of the COMP mutations in the C-type motif of the linker and T3 repeats $[15,16]$. In our study, we identified an autosomal dominant MED in a large Chinese family carry COMP D401N mutation. A single case of D401N mutation was previously reported in a COMP mutation screening study of 100 families in UK [17]. We hypothesize that the mutation identified disrupt calcium binding, as the mutation identified falls in the calmodulin-like domain of $C O M P$, which constitute calciumbinding pockets and its binding to calcium is reported to be essential for the COMP conformation [18]. The previous study show that the disruption of matrix formation and cell-matrix interaction by mutated $C O M P$ on may be a major element in the pathogenesis of COMP-associated chondrodysplasias $[9,11]$.

In conclusion, we identified a missense dominantly inherited recurrent variant in the COMP gene in a large family. This is the first report of COMP D401N mutation in Asian population. We extended the mutation repertoire of COMP gene in patients with MED, paving the way for gene therapy and prenatal diagnosis of MED.

\section{Acknowledgment}

We thank all patients and control individuals for their generous participation in this study. This work is sponsored by the Fund for Shanxi "1331 Project" Collaborative Innovation Center, 1331 CIC (206541001).

\section{References}

1. Bonafe L, Cormier Daire V, Hall C, Lachman R, Mortier G, et al. (2015) Nosology and classification of genetic skeletal disorders: 2015 revision. American journal of medical genetics Part A 167A(12): 2869-2892.

2. Michael D Briggs, PAB, Michael J Wright, Katarzyna A Pirog (2015) New therapeutic targets in rare genetic skeletal diseases. Expert Opin Orphan Drugs 3(10): 1137-1154.

3. Unger S, Bonafe L, Superti Furga A (2008) Multiple epiphyseal dysplasia: clinical and radiographic features, differential diagnosis and molecular basis. Bailli \& Egrave Re S Best Practice \& Research in Clinical Rheumatology 22(1): 19-32.

4. Anthony S, Munk R, Skakun W, Masini M (2015) Multiple epiphyseal dysplasia. The Journal of the American Academy of Orthopaedic Surgeons 23(3): 164-172.

5. Kim OH, Park H, Seong MW, Cho TJ, Nishimura G, et al. (2011) Revisit of multiple epiphyseal dysplasia: Ethnic difference in genotypes and COMParison of radiographic features linked to the COMP and MATN3 genes. American Journal of Medical Genetics Part A 155A(11): 26692680 .
6. Balasubramanian K, Li B, Krakow D, Nevarez L, Ho PJ, et al. (2017) MED resulting from recessively inherited mutations in the gene encoding calcium-activated nucleotidase CANT1. American Journal of Medical Genetics Part A 173(9): 2415-2421.

7. Cho TJ, Kim OH, Lee HR, Shin SJ, Yoo WJ, et al. (2010) Autosomal recessive multiple epiphyseal dysplasia in a Korean girl caused by novel COMPound heterozygous mutations in the DTDST (SLC26A2) gene. Journal of Korean medical science 25(7): 1105-1108.

8. Oldberg A, Antonsson P, Lindblom K, Heinegard D (1992) COMP (cartilage oligomeric matrix protein) is structurally related to the thrombospondins. Journal of Biological Chemistry 267(31): 2234622350.

9. Acharya C, Yik JHN, Kishore A, Van Dinh V, Di Cesare PE, et al. (2014) Cartilage oligomeric matrix protein and its binding partners in the cartilage extracellular matrix: Interaction, regulation and role in chondrogenesis. Matrix Biology 37: 102-111.

10. Hansen U, Platz N, Becker A, Bruckner P, Paulsson M, et al. (2011) A secreted variant of cartilage oligomeric matrix protein carrying a chondrodysplasia-causing mutation (p.H587R) disrupts collagen fibrillogenesis. Arthritis \& Rheumatology 63(1): 159-167.

11. Posey KL, Coustry F, Veerisetty AC, Liu PM, Alcorn JL, et al. (2012) Chop (Ddit3) Is Essential for D469del-COMP Retention and Cell Death in Chondrocytes in an Inducible Transgenic Mouse Model of Pseudoachondroplasia. American Journal of Pathology 180(2): 727-737.

12. Ikegawa S, Ohashi H, Nishimura G, Kim KC, Sannohe A, et al. (1998) Novel and recurrent $\operatorname{COMP}$ (cartilage oligomeric matrix protein) mutations in pseudoachondroplasia and multiple epiphyseal dysplasia. Human Genetics 103(6): 633-638.

13. Chen D, Zhao N, Wang J, Li Z, Wu C, et al. (2017) Whole-exome sequencing analysis of Waardenburg syndrome in a Chinese family. Hum Genome Var 4: 17027.

14. Briggs MD, Bell PA, Pirog KA (2015) The utility of mouse models to provide information regarding the path molecular mechanisms in human genetic skeletal diseases: The emerging role of endoplasmic reticulum stress (Review). International Journal of Molecular Medicine 35(6): 1483-1492.

15. Jackson GC, Mittaz-Crettol L, Taylor JA, Mortier GR, Spranger J, et al. (2012) Pseudoachondroplasia and multiple epiphyseal dysplasia: A 7-year COMPrehensive analysis of the known disease genes identify novel and recurrent mutations and provides an accurate assessment of their relative contribution. Human Mutation 33(1): 144-157.

16. Briggs MD, Brock J, Ramsden SC, Bell PA (2014) Genotype to phenotype correlations in cartilage oligomeric matrix protein associated chondrodysplasias. European Journal of Human Genetics Ejhg 22(11): 1278-1282.

17. Kennedy J, Jackson G, Ramsden S, Taylor J, Newman W, etal. (2005) COMP mutation screening as an aid for the clinical diagnosis and counselling of patients with a suspected diagnosis of pseudoachondroplasia or multiple epiphyseal dysplasia. European journal of human genetics EJHG 13(5): 547-555.

18. Thur J, Rosenberg K, Nitsche DP, Pihlajamaa T, Alakokko L, et al. (2001) Mutations in cartilage oligomeric matrix protein causing pseudoachondroplasia and multiple epiphyseal dysplasia affect binding of calcium and collagen I, II, and IX. Journal of Biological Chemistry 276(9): 6083-6092. 
(C) () This work is licensed under Creative

To Submit Your Article Click Here: Submit Article

DOI: $10.32474 /$ PRJFGS.2018.01.000113

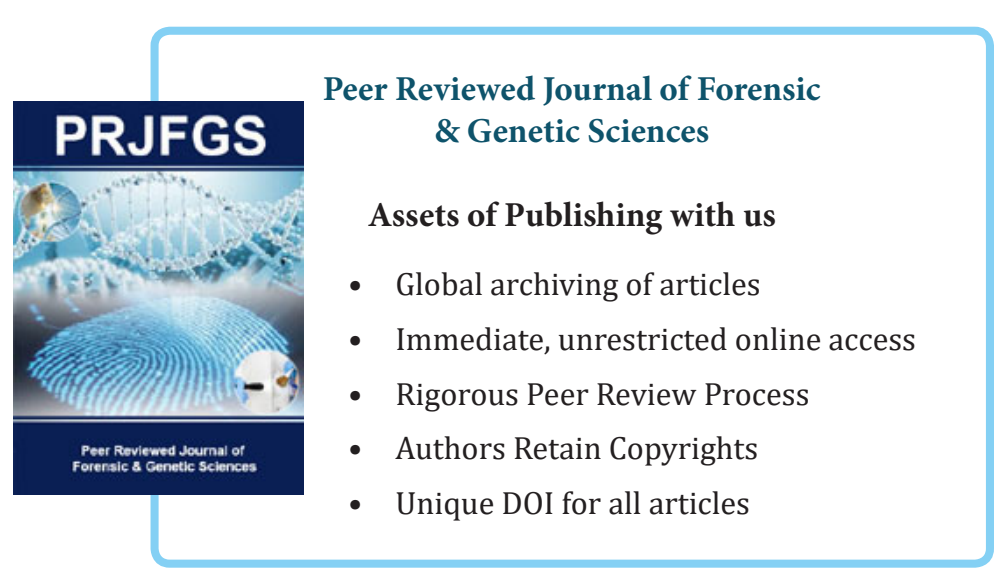

